\title{
Presencia de la mujer y brecha de género en la política local española
}

\author{
ALEJANDRo EsPí-HERNÁNDEZ
}

Universidad de Murcia

doi: https://doi.org/10.20318/femeris.2017.3556

Resumen. Este artículo analiza en términos cuantitativos y cualitativos la presencia actual de la mujer en la administración local española con perspectiva temporal comparada. Con el análisis e interpretación de diferentes datos, pretendemos observar la evolución histórica y estado actual de la paridad de género en la administración local española. Del mismo modo comprobar si las medidas legales y orgánicas tendentes a la corrección de los desequilibrios de género, están consiguiendo mejorar la representación de la mujer en el poder local. El presente trabajo se interesa, además, en conocer el papel de la mujer, respecto de los hombres, una vez que han asumido responsabilidad pública local. Para lograr todos estos fines se analizan aspectos tanto externos (fundamentalmente relativos a concejalías y alcaldías), como internos (distribución de cargos y áreas temáticas, así como promoción interna la mujer), que proyectan una visión comparada de la situación de mujeres y hombres en el conjunto de la administración local española desde que se tienen datos. Variables que nos van a permitir argüir unas conclusiones precisas sobre la igualdad, o ausencia de ella, en el seno de aquellas administraciones públicas que son consideradas las más próximas a la ciudadanía. La interpretación de los datos, fundamentalmente los externos, nos muestran una evolución positiva en el número de concejalas al tiempo que una fuerte resistencia en la figura de la alcaldía. Por su parte, los datos internos, nos alertan de la existencia de una desigualdad y reproducción de los roles de género dentro de los propios ayuntamientos.

Palabras clave: mujer, administración local, concejalas, alcaldesas, paridad, ayuntamientos.

Abstract. This article analyses in quantitative and qualitative terms the current presence of women in the Spanish local administration with a temporary comparated perspective. With the analysis and the interpretation of different data, we try to observe the historical evolution and the actual state of gender parity in the Spanish local administration. In the same way, we pretend to verify if legal and organic actions leading to correct gender imbalances are improving the representation of women in local power. The present work is also interested in knowing the role of women, regarding men, once they have assumed local public responsibility. In order to achieve all of these aims, we analyse external aspects (mainly concerning councils and mayors), as well as internal ones (distribution of positions and thematic areas, as well as internal promotion of women) which project a compared view of the situation of women and men in the Spanish local administration since they have data. These variables will allow us to argue precise conclusions about the equality, or absence of it, within those public administrations that are considered the closest ones to citizenship. The interpretation of the data, mainly the external ones, shows us a positive evolution in the number of councilors and, at the same time, a strong resistance in the figure of the mayor's office. On the other hand, the internal data alert us about the existence of an inequality and reproduction of the gender roles within the own municipalities..

Keywords: woman, local administration, councilors, mayors, parity, city councils.

**alejandro.espih@um.es 


\section{Introducción}

Durante siglos, los hombres han dominado la dirección política de nuestro país en todos sus niveles, recayendo exclusivamente en ellos la responsabilidad de asumir las riendas de la nación en prácticamente todos los periodos históricos desde la llegada del sufragio universal. Un símil político a la situación histórica que la mujer ha atravesado en todas las esferas de la sociedad respecto del hombre: sometimiento, inferioridad y discriminación. Como señala Rosa Cobo (2002), las mujeres constituyen un colectivo marginado y subordinado en todas y cada una de las sociedades existentes. En consecuencia, así ha venido ocurriendo en la dirección de los asuntos públicos y organizaciones políticas.

Con la extensión del sufragio activo y pasivo a la mujer en la práctica totalidad del mundo hace relativamente pocos años ${ }^{1}$, fueron consolidándose las democracias de mediados y finales de siglo XX, garantes de la igualdad en sus marcos normativos. Ahora bien, resulta preciso detenernos en un análisis minucioso para conocer si esa igualdad normativa, la igualdad formal, es también efectiva o se aproxima a ello. En este caso, nos interesa conocer la participación de la mujer en la representación política local de nuestro país, dado que es considerada la administración más próxima a la ciudadanía.

Cuando en el presente hablamos de mujer e igualdad, suele emanar el falso mito conocido como "espejismo de la igualdad"; creencia de que aquella ya está conseguida. Ese espejismo aparece igualmente cuando trabajamos sobre mujer y poder, ámbito que nos concierne en este trabajo, en tanto que hay una creencia extendida de que, dado el incremento de los niveles educativos de la mujer, su inclusión en el mercado laboral y algunos avances en los roles de género, la participación de las mujeres en la vida pública ya ha debido cosechar los niveles aceptados. Es decir, que no existe ningún problema de desigualdad en el acceso y permanencia a los cargos públicos de la administración local española. Esta idea es consecuencia directa de la falta de conocimiento sobre datos que reflejen la situación de la paridad de género en el municipalismo español, que distan significativamente de la percepción que pueda tenerse al respecto.

De hecho, si algo motivará el nacimiento de la Ley 3/2007, entre otras cuestiones, será la desequilibrada participación de mujeres y hombres en las instituciones públicas y partidos políticos de nuestro país. El legislador es consciente de la necesidad de integrar la igualdad de manera transversal en todas las políticas y decisiones, a fin de promocionar a la mujer, visibilizarla y ascenderla al escalón de partida donde ya se encuentran los hombres por el hecho de serlo. El contenido de la 3/2007 incide en la inclusión de la mujer en las listas electorales a fin de lograr el acceso a cargos públicos, en tanto que mitad de la población que se ve históricamente discriminada en este sentido.

Normalmente, los estudios que versan sobre la mujer en política, no suelen tratar con asiduidad la participación de la misma en la administración local, centrándose aquellos, mayormente, en análisis focalizados a parlamentos nacionales y/o autonómicos, así

\footnotetext{
${ }^{1}$ En España se reconoce el sufragio femenino en 1931, votando las mujeres por primera vez en las elecciones a Cortes de noviembre de 1933.
} 
como en los liderazgos femeninos y el peso orgánico de las mujeres en las organizaciones políticas. Con este trabajo pretendemos ampliar el campo de conocimiento político y social relativo a la presencia y participación de la mujer en el municipalismo español.

\section{Hipótesis iniciales}

En el año 2015 España celebró en todo su territorio nacional elecciones municipales y, en algunas autonomías, elecciones a los entes territoriales de carácter autonómico, de acuerdo a la Ley Orgánica 5/1985 de Régimen Electoral General. Con la celebración de estos comicios para los más de 8.000 ayuntamientos del país, el mapa político municipal ha atravesado una modificación partidista que abarca desde numerosas alcaldías hasta las propias autonomías, pasando por el poder provincial. Sin embargo, nos resulta de mayor interés analizar los datos relativos a la situación de la mujer en los consistorios españoles, para comprobar si, además de cambios de gobierno, se han producido notorias mejoras en lo referente a la brecha de género existente en nuestro país, en cuanto a presencia, representación y atribución de responsabilidades de las mujeres en los cargos públicos municipales.

Nuestro objetivo, por consiguiente, es conocer la evolución de la paridad de género en la administración local española desde varios enfoques y en diferentes momentos temporales de la presente democracia. Partimos para esta investigación considerando como hipótesis principal (H1) que el poder local español continúa siendo predominantemente masculino, y que, (H2) las disposiciones orgánicas de los partidos y las medidas legales introducidas para corregir el desequilibrio de género en el poder local, especialmente las contenidas en la citada Ley Orgánica 3/2007 para la igualdad efectiva de mujeres y hombres, han conseguido mejoras significativas en los datos, aunque todavía se encuentran lejos de lograr una paridad real.

\section{Metodología}

Para la comprobación de las hipótesis, este artículo efectúa un recorrido temporal cuantitativo por los diferentes datos que nos van a ilustrar" la situación de la brecha de género en las instituciones públicas españolas, concretamente las de ámbito local, desde que se tienen datos hasta el año 2015, prestando especial interés al impacto de la Ley Orgánica $3 / 2007$, para la igualdad efectiva de mujeres y hombres. Realizamos un análisis tanto externo (evolución de la incorporación de la mujer en concejalías y alcaldías) como interno (reparto de áreas temáticas en los consistorios, de cargos y promoción de la mujer de una legislatura a la siguiente). Todo ello sobre las entidades públicas que son consideradas la más cercanas a la ciudadanía y, por ende, aquellas que pueden visibilizar de manera más eficaz a la mujer, contribuyendo así a la normalización de género desde el espacio común al conjunto de la sociedad: lo público. Mediante la lectura e interpretación de los datos 
podremos llegar a conclusiones finales que responden directamente a las hipótesis de trabajo inicialmente esbozadas.

Los datos que van a ser tratados son extraídos de diferentes administraciones y organismos públicos como la Federación Española de Municipios y Provincias (FEMP), el Instituto de la Mujer y los Ministerios de Hacienda y Administraciones Públicas, así como el Ministerio del Interior.

\section{Resultados}

\subsection{Análisis externo. Más concejalas, más alcaldesas, pero todavía lejos de la paridad}

Los términos democracia y participación ciudadana están necesariamente conectados entre sí, en tanto que el primero requiere del segundo para su plena efectividad. El reconocimiento democrático de la ciudadanía política conlleva no sólo el goce de derechos civiles como son el derecho al sufragio activo y pasivo, las libertades civiles o la elegibilidad, sino también el deber de participar en la vida política. He aquí donde entra en disposición el artículo 21 de la Declaración Universal de los Derechos Humanos, que reconoce el derecho de toda persona a participar en el gobierno de su país, la igualdad de acceso de hombres y mujeres al poder, la toma de decisiones y el liderazgo en todos los niveles. Las mujeres han librado numerosas contiendas para la consecución de sus derechos políticos, pero, sin embargo, la paridad entre hombres y mujeres en la vida política no es, todavía, una realidad. Las mujeres permanecen infra-representadas en la toma de decisiones en general y en los órganos de decisión local en particular (García, 2011).

Efectivamente, el poder no se tiene, se ejerce: no es una esencia o una sustancia, sino una red de relaciones cuyo espacio natural donde se fraguan los pactos patriarcales es la política (Varela, 2013). Con los resultados que a continuación comenzamos a detallar, pretendemos visibilizar, por tanto, a quién ejerce el poder en el ámbito municipal en nuestro país desde que la democracia echó a andar o bien desde que se tienen datos, para poder esgrimir conclusiones desde una perspectiva de género.

En España, con la entrada en vigor de la citada Ley Orgánica 3/2007, de 22 de marzo, se introdujo la obligatoriedad de que los partidos políticos presentaran listas electorales cerradas y bloqueadas con una composición equilibrada de hombres y mujeres, aplicando un suelo mínimo de un 40\% de representación por parte de alguno de los géneros y un máximo de $60 \%$ para el otro ${ }^{2}$. Con esta medida legal se dispone, desde entonces, a los partidos políticos al servicio de la igualdad, al margen de las políticas que en este sentido decidan aplicar a nivel orgánico cada uno de ellos, como es el caso de la regla del 50-50\% que aplica Izquierda Unida desde 2012 o las listas cremalleras que presenta el PSOE desde 2014, entre otras. En definitiva, suponen acciones positivas que pretenden lograr que

\footnotetext{
${ }^{2}$ Estas disposiciones no afectan a los municipios menores de 5000 habitantes, lugares donde más se incrementa la brecha de género como más adelante observaremos.
} 
todo el mundo parta de la misma línea de salida para que cada cual llegue donde le permitan sus capacidades.

\subsubsection{Concejalías}

Ha sido en el año 2015 cuando las mujeres en España han conseguido superar, por primera vez en la presente democracia, el umbral del 35\% de las concejalías totales de nuestro país, la cifra más alta de toda la serie histórica. Ciertamente, como observamos a continuación en el gráfico I, la representación de la mujer en los consistorios municipales aumenta progresivamente tras la celebración de cada elección municipal, lo que supone un dato de entrada positivo, aunque todavía insuficiente en vista a que los hombres ocupan todavía hoy el $64,43 \%$ de las actas de concejal totales. A pesar de la satisfacción que podría producirnos este aumento de presencia femenina en los ayuntamientos, es relevante destacar que entre 2007 y 2015, horquilla de ocho años, el porcentaje de concejalas en ayuntamientos españoles apenas ha aumentado un 5,07\%, dato todavía muy discreto que demuestra la paulatina evolución al respecto.

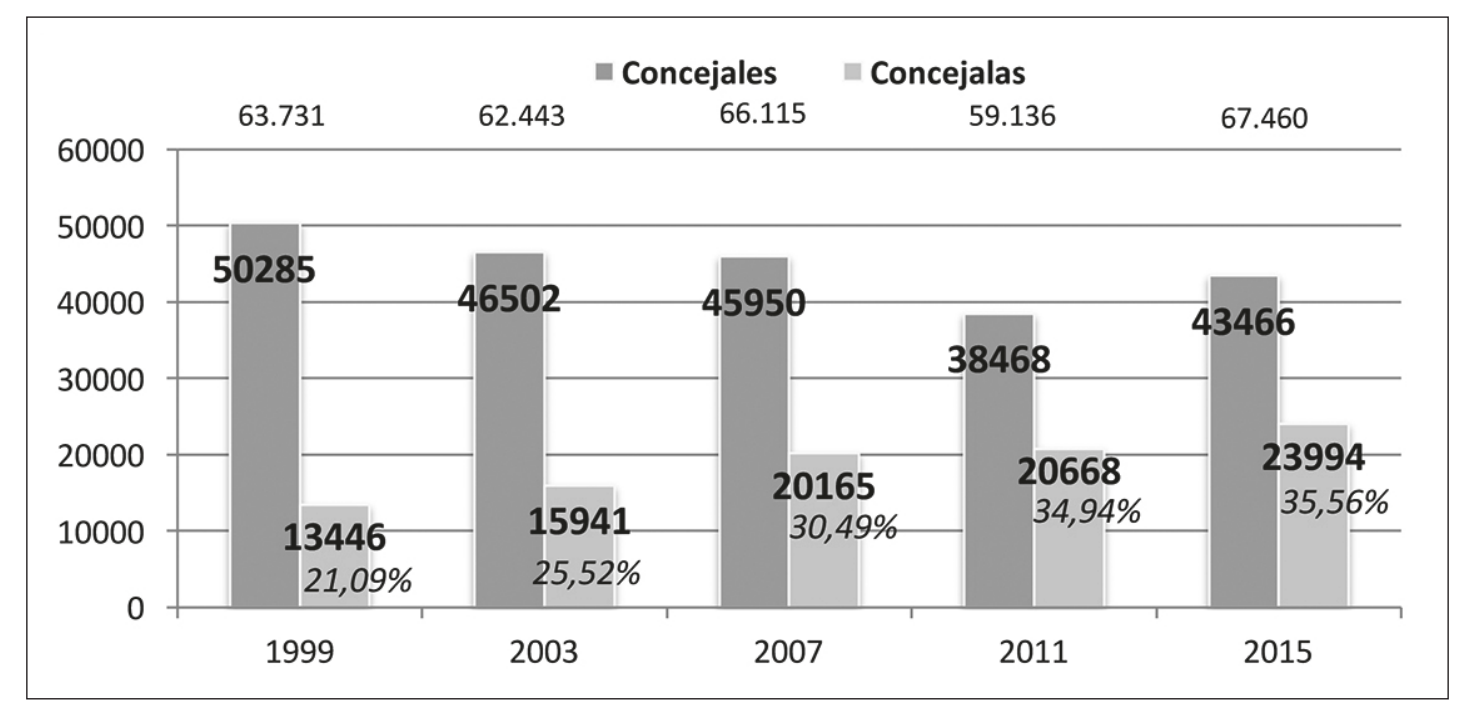

Gráfico 1. Evolución del número de concejales y concejalas en España desde 1999.

Fuente: Elaboración propia a partir de los datos del Instituto de la Mujer y para la Igualdad de Oportunidades elaborados, a su vez, en base a los datos facilitados por el Ministerio de Hacienda y Administraciones Públicas.

Tal y como puede apreciarse en estos datos, se ha producido un aumento notable del número de concejalas en España respecto de los varones, con un repunte de concejales hombres en 2015 que parece atender mayormente a un aumento de concejalías totales en el país respecto a $2011^{3}$. Así, en el año 2003 las mujeres con acta de concejala apenas

\footnotetext{
${ }^{3}$ En el año 2011 se eligieron 59.136 concejales/as mientras que en 2015 han sido 67.460 las actas expedidas.
} 


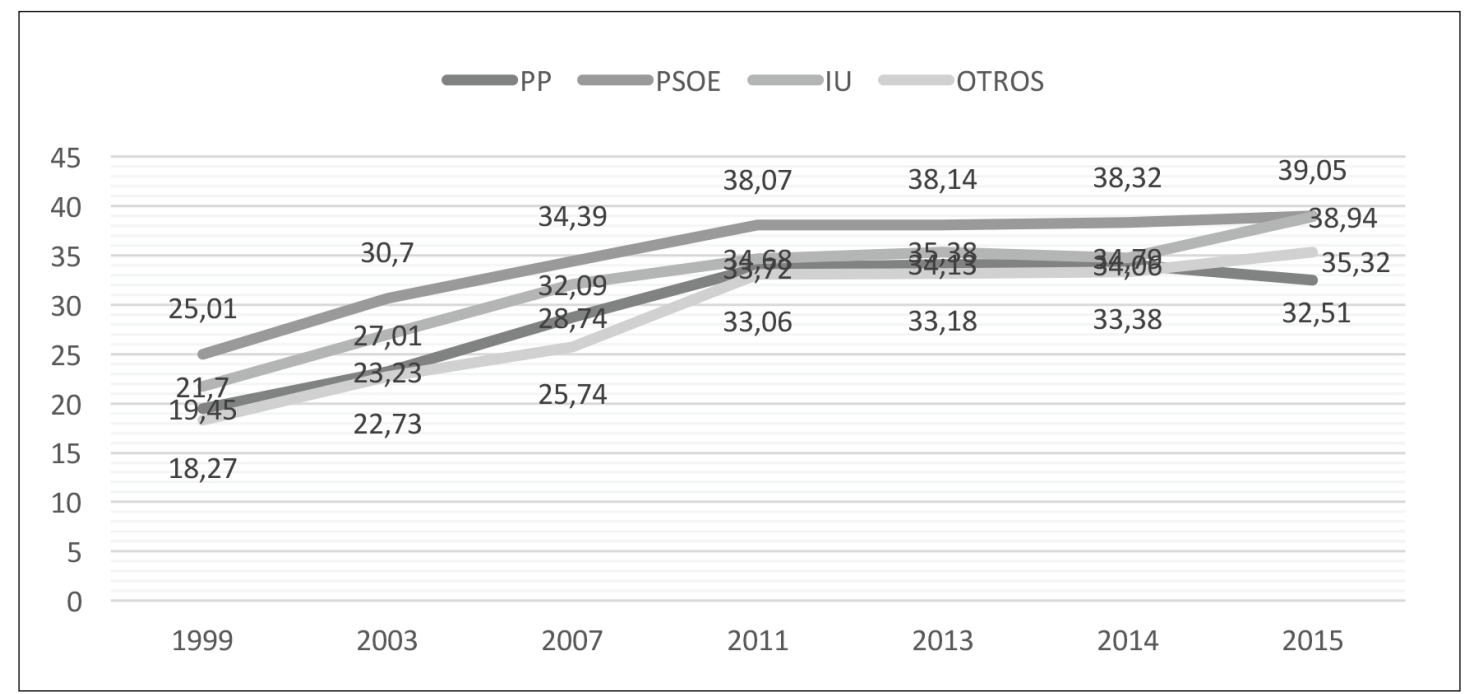

Gráfico 2. Evolución del número de concejalas por partidos políticos en España desde 1999 a 2015.

Fuente: Elaboración propia a partir de los datos del Instituto de la Mujer y para la Igualdad de Oportunidades elaborados, a su vez, en base a los datos facilitados por el Ministerio de Hacienda y Administraciones Públicas.

constituían el 25,53\% del total, aumentando hasta el 30,75\% en 2007 con la aplicación de la Ley de Igualdad, lo que supuso un incremento de 4.224 concejalas, la mayor subida de toda la serie histórica. En 2015 se ha logrado nuevamente el máximo histórico en España con 23.994 concejalas $(35,57 \%)$, cifra que todavía continúa lejos de una paridad que podría ser "aceptada" en las barreras aceptadas del 60-40\% referidas en la legislación.

Si cruzamos la variable de las concejalías con la variable partidos políticos nacionales, observamos que el partido que ha presentado mayor cifra de edilas, es el PSOE, muy seguido de Izquierda Unida, ambos por encima del Partido Popular en todos los años marcados como referencia. No obstante insistimos en la leve evolución que se ha producido al respecto, pues en dieciséis años, tan solo en el mejor de los casos (el del Partido Socialista), se evoluciona del 25,01\% al 39,05\% de representación femenina 4 .

\subsubsection{Las alcaldías en España, foco de resistencia masculina}

Sin embargo, donde todavía pueden apreciarse los notables síntomas de masculinización del poder local en España, es en la ostentación de alcaldías existentes en nuestro país. En este sentido, las diferencias porcentuales por sexos denotan la leve apuesta por alcaldables mujeres por parte de los partidos políticos españoles, incidiendo así en el sesgo de género para la más alta responsabilidad municipal. Si bien es preciso recordar que la ley de igualdad no prioriza género para encabezar listas electorales, dejando a instancia de cada partido esta designación. Los datos de la tabla I nos muestran un ligero aumento del número de alcaldesas en España en toda la trayectoria democrática, mejorando en el

${ }^{4}$ Sólo se tienen datos al respecto desde 1999. 
periodo temporal de 32 años (de 1983 a 2015), en tan solo 17 puntos porcentuales. En el año 1983 el número de alcaldesas era de 164 (un 2,04\% de las alcaldías totales), constituyendo en la actualidad el 19,09\% (1550 alcaldesas), que supone un máximo histórico. El incremento es notorio, pero insuficiente, pues el número de alcaldes en la actualidad multiplica casi por seis el número de alcaldesas.

\begin{tabular}{|c|c|c|c|}
\hline Año & Número de alcaldesas & \% sobre total & Incremento \\
\hline 1983 & 164 & $2,04 \%$ & 1,18 \\
\hline 1987 & 249 & $3,22 \%$ & 1,64 \\
\hline 1991 & 393 & $4,86 \%$ & 1,67 \\
\hline 1995 & 529 & $6,53 \%$ & 3,08 \\
\hline 1999 & 779 & $9,61 \%$ & 2,95 \\
\hline 2003 & 1014 & $12,56 \%$ & 2,04 \\
\hline 2007 & 1179 & $14,60 \%$ & 2,5 \\
\hline 2011 & 1355 & $17,1 \%$ & 0,07 \\
\hline 2013 & 1393 & $17,17 \%$ & 1,92 \\
\hline $2015 *$ & 1550 & $19,09 \%$ & $\mathbf{1 7 , 0 5 \%}$ \\
\hline Total & - & - & \\
\hline
\end{tabular}

* Dato a de 30 de noviembre de 2015

Tabla I. Evolución del número de alcaldesas en España.

Fuente: Elaboración propia a partir de los datos del Instituto de la Mujer y para la Igualdad de Oportunidades elaborados, a su vez, en base a los datos facilitados por el Ministerio de Hacienda y Administraciones Públicas.

Observando el gráfico 3, podemos contemplar una tendencia en el tiempo de incorporación de la mujer en las alcaldías españolas, que no obstante relata un ritmo ascendente muy leve. Ello nos hace continuar poniendo el foco de atención y análisis en el exceso de masculinización de las alcaldías españolas, que presentan una distribución por sexos de un 80\%-20\%, dato que ni de lejos se aproxima a los mínimos de 60-40 establecidos por ley para la confección de listas electorales. No obstante, no podemos dejar de mencionar que los dos ayuntamientos más grandes de España, Madrid y Barcelona, están desde junio de 2015 presididos por mujeres: Manuela Carmena y Ada Colau, respectivamente. Hecho relevante para destacar, aunque insuficiente en sí mismo. La mujer permanece altamente invisibilizada en la figura política de la alcaldía en España (ver gráfico 3).

La Ley $3 / 2007$, a tenor de los datos anteriores, no ha provocado un impacto significativo en el número de alcaldías ostentadas por mujeres, pues la evolución de las cifras mantiene un ritmo similar año tras año. Es preciso recordar, nuevamente, que la ley no establece ningún tipo de priorización de género para encabezar listas electorales de cara a la alcaldía, quedando esta decisión en manos de los propios partidos políticos y sus mecanismos para esta elección o nombramiento. A su vez, las cuotas de género de los propios partidos políticos tampoco parecen incidir en esta cuestión. Todas ellas aluden a cómo la mujer debe ser incorporada en las listas electorales; encabezarlas parece atender a otras cuestiones. 


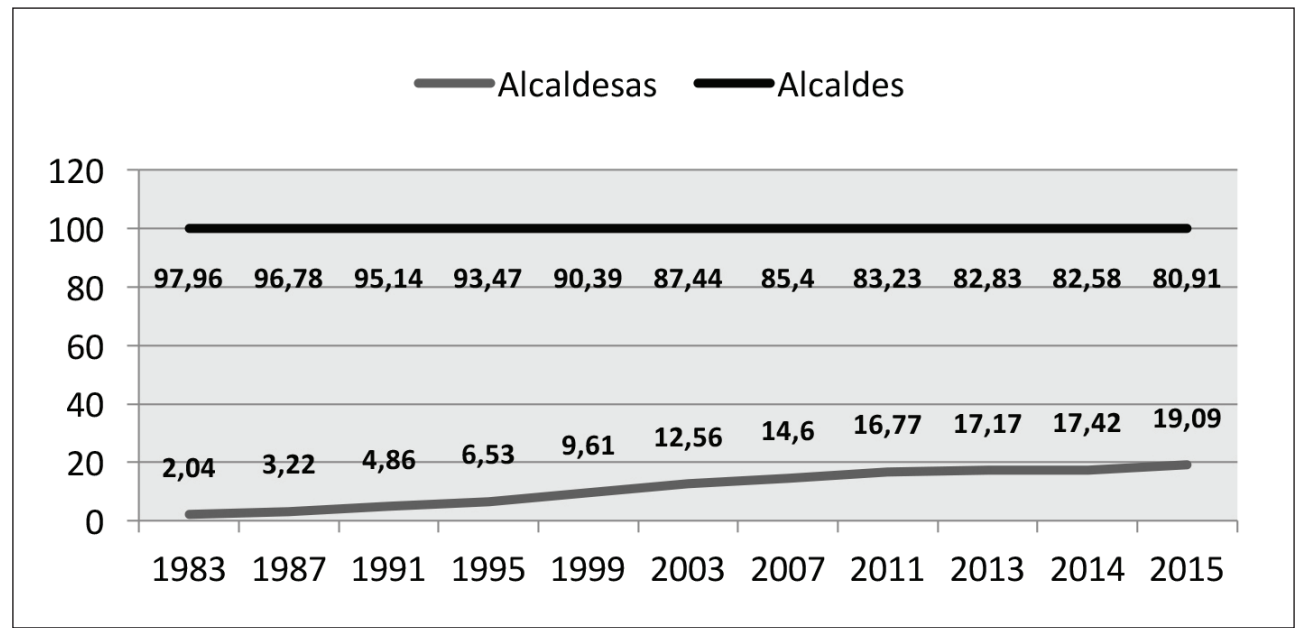

Gráfico 3. EEvolución del número de concejalas por partidos políticos en España desde 1999 a 2015.

Fuente: Elaboración propia a partir de los datos del Instituto de la Mujer y para la Igualdad de Oportunidades elaborados, a su vez, en base a los datos facilitados por el Ministerio de Hacienda y Administraciones Públicas.

Por consiguiente, estamos en disposición de afirmar que existe un claro "filtro" para las mujeres en el seno de los partidos políticos españoles para acceder a las alcaldías, en relación al porcentaje de concejalas electas en los mismos partidos. Esta afirmación la podemos corroborar mediante la interpretación de los datos de la Tabla II, donde cruzamos concejalías y alcaldías por partidos. Además, nos permite vislumbrar cómo la cifra más alta de alcaldesas de un mismo partido político en 2015 en España no llega ni al 30\% de sus alcaldías totales logradas, situándose el índice histórico más elevado en un 40,98\% conseguido por el PSOE en el año 2007 con 1.193 alcaldesas de un total de 2.911 alcaldías a las que accedió. El diferencial de la tabla II ilustra nuevamente las remarcadas diferencias porcentuales entre ambos tipos de cargos públicos ${ }^{5}$ a los que nos venimos refiriendo.

\begin{tabular}{|l|c|c|c|c|c|}
\hline \multicolumn{1}{|c|}{ Partido } & \% Concejalas & Número & \% Alcaldesas & Número & Diferencial \\
\hline PP & $32,51 \%$ & 7382 & $16,53 \%$ & 506 & $-15,98 \%$ \\
\hline PSOE & $39,05 \%$ & 7639 & $22,08 \%$ & 553 & $-16,97 \%$ \\
\hline IU & $38,94 \%$ & 803 & $18,49 \%$ & 27 & $-20,45 \%$ \\
\hline Otros & $35,32 \%$ & 8170 & $18,69 \%$ & 293 & $-16,63 \%$ \\
\hline
\end{tabular}

Tabla II. Número de concejalas y alcaldesas por partidos políticos en 2015

Fuente: Elaboración propia a partir de los datos del Instituto de la Mujer y para la Igualdad de Oportunidades elaborados, a su vez, en base a los datos facilitados por el Ministerio de Hacienda y Administraciones Públicas.

La evolución del número de alcaldesas en España desde 1999 hasta 2015 por partidos políticos, refuerza la hipótesis de los avances en la paridad en el poder local, pero siguen

${ }^{5}$ No descuidamos que las alcaldesas son también concejalas. 
denotando una vez más la existencia de un proceso todavía muy paulatino que proyecta una resistencia a la apuesta por la mujer para la ostentación de las alcaldías. Cabe destacar que el número de alcaldesas de los dos principales partidos políticos en 2007, PP y PSOE, llegaron a rozar el $40 \%$ de sus alcaldías totales. Se trata de un dato excepcional en la serie histórica que duplica al número de alcaldesas actuales de los mismos partidos y que no ha vuelto a sucederse. Tras los últimos comicios de 2015, el PSOE es el partido que cuenta con mayor número de alcaldesas (22,08\%), seguido de IU y “otros" con 18,49\% y 18,69\% respectivamente. Un poco atrás queda el PP que cuenta con el 16,53\% de alcaldesas.

En síntesis, y como refleja el gráfico 4, la participación política de la mujer en la administración local española se ha incrementado ligeramente desde las primeras elecciones locales (1979) hasta las últimas (2015). Sin embargo, la leve mejora denota una todavía resistencia a la incorporación de la mujer, que entra paulatina y tardíamente en el poder local. Es preciso recordar que la participación femenina en la vida política y pública se incrementa no sólo por las medidas legales, sino, además, como citábamos anteriormente, por las decisiones orgánicas de los partidos como la puesta en marcha de cuotas de género u otras disposiciones similares. Las cuotas de representatividad femenina para los cargos orgánicos y públicos en los partidos comenzaron a aparecer en España a finales de los años ochenta. El PCE introdujo sus medidas de discriminación positiva en 1987, siendo en 1988 cuando el PSOE puso en marcha su cuota femenina del 25\%. Ya en los años 90, IU aumentó la cifra en su partido al 35\%. En 1997, PSOE e IU elevan la cuota a un máximo de $60 \%$ y un mínimo de 40\% para cualquier género. En el caso del Partido Popular, no se llega a articular ninguna cuota de género hasta el momento (Verge, 2006). En el año 2006, previo a la aprobación de la ley de igualdad, eran 5 partidos políticos españoles los que ya habían adoptado cuotas voluntarias de género con el objetivo de equilibrar la composición por sexos de sus candidaturas (Davidson-Schnuch, 2006).

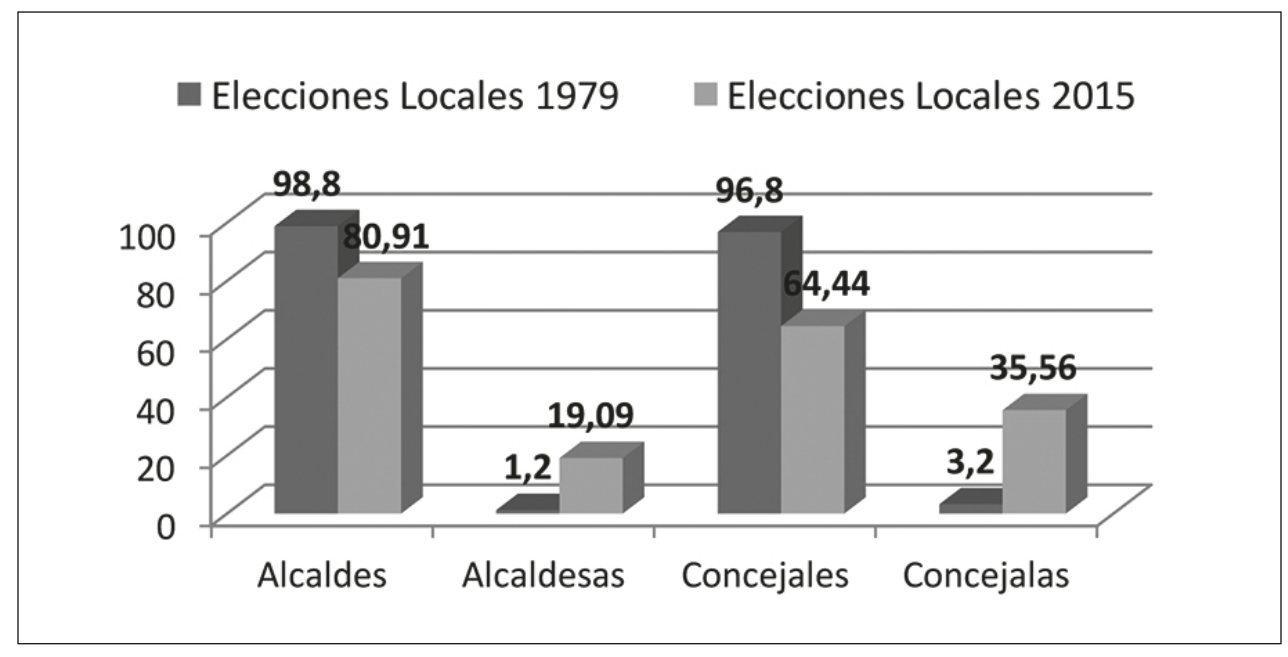

Gráfico 4. Comparativa del porcentaje de alcaldías y concejalías por género en España, 1979 y 2015.

Fuente: Registro de Representantes electos del Ministerio de Administraciones Públicas. Elaboración propia. 
En el gráfico 4, como síntesis a este apartado, ilustramos los datos comparativos de las primeras (1979) y últimas (2015) elecciones municipales en España tanto para alcaldías como concejalías por género.

\subsubsection{El factor dimensional de los municipios en la brecha de género}

El tamaño de los municipios españoles es una variable trascendental para comprender la brecha de género existente en el poder político local que venimos ilustrando con la lectura de los diferentes datos de concejalías y alcaldías. Los más de 8.000 municipios existentes en España presentan una división administrativa local muy heterogénea, coexistiendo municipios minúsculos frente a grandes urbes. En relación a la brecha de género en el poder local español, encontramos una tendencia consistente en que, a medida que el municipio disminuye de tamaño, la brecha de género aumenta. Es decir, la paridad de género en el municipalismo mejora a medida que el tamaño del municipio es mayor. Esta tendencia denota la existencia de una importante resistencia a la inclusión de la mujer en la política local proveniente fundamentalmente por las zonas rurales.

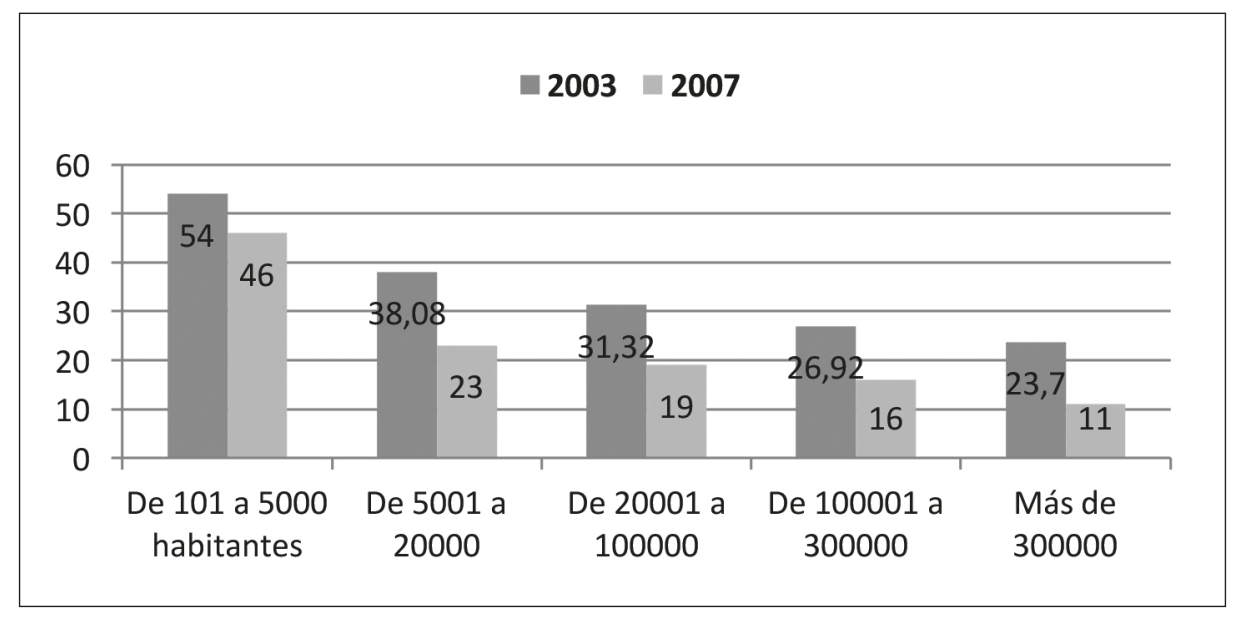

Gráfico 5. Brecha de género (\%) en el municipalismo en España según dimensión del municipio en 2003 y 2007.6

Fuente: Estudio Impacto de la Ley Orgánica 3/2007, para la Igualdad Efectiva de Mujeres y Hombres en la Representación de Mujeres en el Ámbito de la Política Local. Elaboración propia.

Los datos del Estudio Impacto de la Ley Orgánica 3/2007, para la Igualdad Efectiva de Mujeres y Hombres en la Representación de Mujeres en el Ámbito de la Política Local de la FEMP (ver gráfico 5), nos permite observar una leve mejora de los años 2003 a 2007 (antes y después de la Ley 3/2007) en cuanto a la presencia de las mujeres en la política local, incluso entre los municipios menores de 5000 habitantes, exentos de aplicación de la Ley 3/2007. Precisamente los municipios comprendidos entre 101 y 5000 habitantes,

${ }^{6}$ No existen datos agregados posteriores. 
existe una alta brecha de género de entorno al 50\%. Por el contrario, aquellas urbes de más de 300.000 habitantes presentaron en 2007 una brecha de género en representación local del $11 \%$. No podemos olvidar que el tamaño del municipio es un factor muy influyente en la magnitud de la brecha de género. Los datos de la Federación ilustran cómo a medida que el tamaño del municipio es mayor, la brecha de género va disminuyendo. Dicho de otro modo, en los municipios de menor tamaño persisten de manera considerable los estereotipos de género, haciendo más difícil el acceso de la mujer a la responsabilidad municipal respecto de las urbes más grandes, que son más propensas a la integración de la mujer en la vida política.

\subsection{Análisis interno}

\subsubsection{Distribución de áreas temáticas en los municipios según género}

Otra de las variables a atender cuando tratamos de analizar la presencia de la mujer en la vida política local, es la relativa a la responsabilidad que asumen en tanto que cargos públicos en perspectiva comparada con los hombres. La distribución de las concejalías en España, por áreas temáticas, nos plasma nuevamente una desigualdad que hunde su motivación a los estereotipos de género y la visión machista de la sociedad. El hecho de que las mujeres consigan actas de concejalas no conlleva, necesariamente, la superación de la brecha de género que venimos analizando, pues aparece otra desigualdad a posteriori no menos importante y que merece su especial atención.

A tenor de los datos ilustrados en el gráfico 6, se puede observar una clara segregación de responsabilidades en la administración local que atiende también a motivación de género. Así, existen claras áreas municipales masculinazadas como son, fundamentalmente Economía y Hacienda, Medio Ambiente, Promoción Económica y Empleo, Régimen

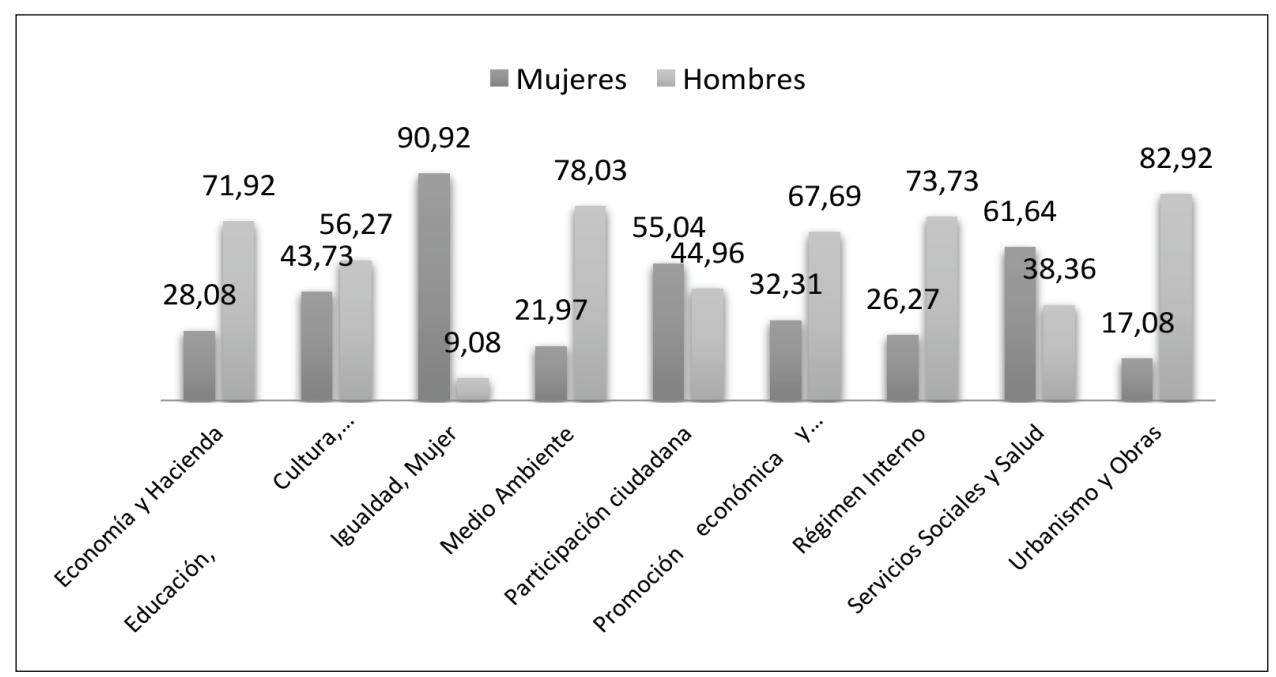

Gráfico 6. Distribución de concejalías en España por sexo, año 2007.

Fuente: Estudio Impacto de la Ley Orgánica 3/2007, para la Igualdad Efectiva de Mujeres y Hombres en la Representación de Mujeres en el Ámbito de la Política Local. Elaboración propia. 
Interno y, por encima de todas, Urbanismo y Obras Públicas. En contra, observamos la existencia de algunas áreas locales claramente feminizadas; son Igualdad, Mujer, Participación Ciudadana, Servicios Sociales y Salud. En una situación algo más equilibrada se halla el bloque de Educación, Cultura, Juventud y Deportes. No obstante, esta última (deportes) también es un área masculinizada pero que, al entrar en contacto en este estudio junto a las otras áreas, especialmente Educación y Cultura, equilibra la balanza.

El rol de género permanece muy visible igualmente en la gestión pública. A las mujeres se las responsabiliza fundamentalmente de las áreas vinculadas al cuidado y el desarrollo de políticas para su avance social, mientras que los hombres asumen las áreas municipales asociadas directamente con la gestión económica y desarrollo del municipio. Se ilustra, por consiguiente, la existencia de una desigualdad que se haya enquistada en la visión androcéntrica de la realidad que los actores políticos, y la sociedad en su conjunto interiorizamos y desarrollamos. El patriarcado no sólo parece evitar la incorporación de la mujer en la administración local, sino que además cuando lo hace, le adjudica responsabilidades que preservan la esencia de los roles que la sociedad les atribuye por el hecho de ser mujer.

\subsubsection{Distribución de cargos y órganos de toma de decisiones en los municipios}

En este análisis sobre la situación externa e interna de la mujer en la administración local española, podemos dar un paso más e incluso observar cuántas mujeres, respecto a hombres, forman parte de los diferentes grados de responsabilidad que en un ayuntamiento pueden asumirse o ser partícipes de. Hablamos de concejalías delegadas, tenencias de alcaldía, concejalías en sí, juntas de gobierno, vicealcaldías y alcaldías. En ese mismo orden se presentan tales responsabilidades y órganos de menor a mayor relevancia en cuanto a poder de decisión en el seno de un consistorio.

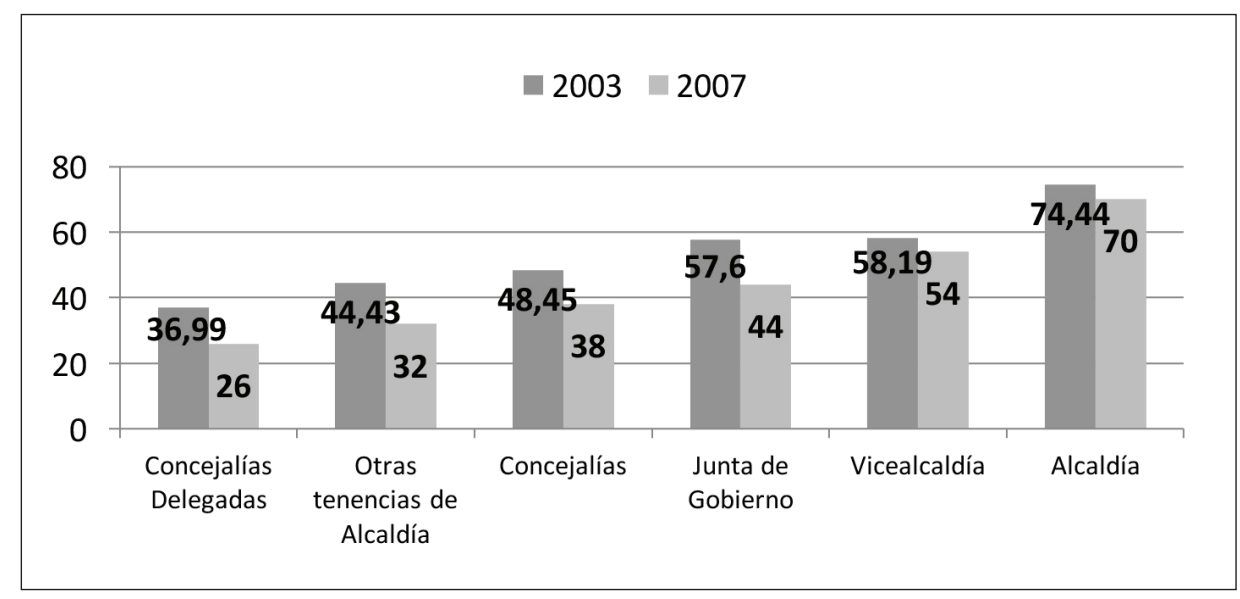

Gráfico 7. Brecha de género (\%) en las diferentes esferas de decisión de los municipios en 2003 y 2007

Fuente: Estudio Impacto de la Ley Orgánica 3/2007, para la Igualdad Efectiva de Mujeres y Hombres en la Representación de Mujeres en el Ámbito de la Política Local. Elaboración propia. 
La brecha de género que existe en estas responsabilidades y órganos de decisión va en aumento a medida que la responsabilidad que se puede adoptar es mayor. Dicho con otras palabras, cuanta mayor responsabilidad se asume en un ayuntamiento, menos mujeres ostentan esa potestad. Con mayor detenimiento podemos comprobarlo en el gráfico 7, que compara los años 2003 y 2007. Nuevamente no disponemos de los datos relativos a 2011 y 2015, que probablemente presenten unas cifras en la misma tendencia, aunque previsiblemente ligeramente corregidos.

Las alcaldías en España presentan la mayor brecha de género de las diferentes categorías de responsabilidad dadas en un Ayuntamiento, como pudimos ver con anterioridad y como se refleja en los gráficos 2 y 7 . Aunque los datos mejoran levemente respecto del año predecesor, la situación sigue ilustrando un escenario de desigualdad dentro de la propia brecha de género ya dada en la administración local. Dicho con otras palabras, nos encontramos con una desigualdad dentro de la desigualdad, valga la redundancia.

\subsubsection{Permanencia y promoción de las mujeres en política local}

A tenor de los datos que presenta el informe de la FEMP, en el año 2007 del total de concejales y concejalas que permanecen ocupando su acta respecto al año 2003, el 77\% fueron hombres. De igual modo, esa repetición es mucho más acusada cuando se trata de las alcaldías, donde las mujeres tan solo repiten, en el ejemplo propuesto del año 2007, en un $11 \%$, mientras que los hombres lo hacen en un $89 \%$. No obstante, en todas las escalas de poder o decisión municipal que plasma el informe, la repetición del hombre respecto a la legislatura anterior es bastante superior al de la mujer.

Además, según otro estudio de la propia Federación Española de Municipios y Provincias (FEMP), en el año 2011, respecto a 2007, renovaron su acta de concejala el 55,2\% de las mujeres, lo que supuso poco más de la mitad de las mismas. El resto, hasta el 100\% de las concejalas en 2011, fueron de nueva incorporación (ver gráfico 8).

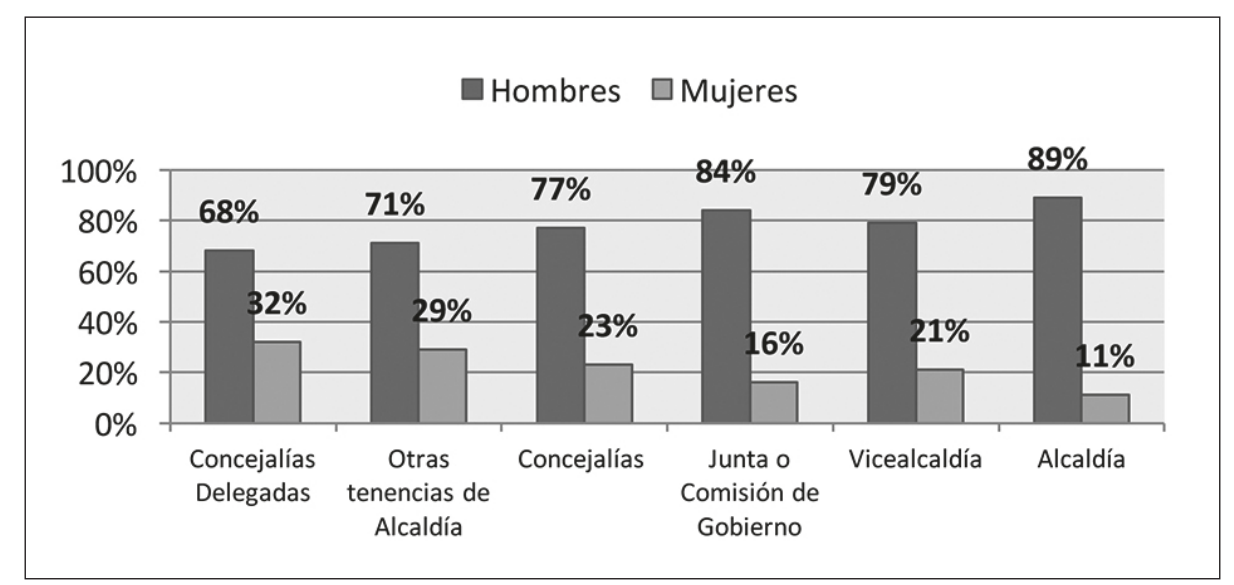

Gráfico 8. Permanencia de mujeres y hombres en cargos públicos en 2007 respecto a 2003.

Fuente: Estudio Impacto de la Ley Orgánica 3/2007, para la Igualdad Efectiva de Mujeres y Hombres en la Representación de Mujeres en el Ámbito de la Política Local. Elaboración propia. 


\section{Conclusiones}

Con los datos que hemos ilustrado en este trabajo, observamos todavía ausencia de presencia equilibrada de mujeres y hombres en los centros de decisión local, pese a que la mujer constituya hoy más del $50 \%$ de la población estatal. La presencia de la mujer en el poder local español es discreta, aunque las cifras se han multiplicado en las últimas décadas, fundamentalmente desde la entrada en vigor de la Ley 3/2007. No obstante, a pesar de los ligeros cambios producidos en estos años de funcionamiento democrático, la igualdad entre mujeres y hombres en la participación política local es todavía un claro objetivo a conseguir.

Como impresión concluyente de lo analizado podemos extraer que, cuando se trata de elegir un número elevado de representantes públicos (concejales/as) la paridad aumenta. Sin embargo, cuando se trata de altos cargos unipersonales (alcaldías), la paridad disminuye. Ello denota la todavía masculización del poder local y la existencia de una mayor promoción y experiencia política de los hombres que llegan al poder respecto a las mujeres. Así mismo refleja que, cuando se debe pasar el filtro de la Ley 3/2007 (primer caso), mejora la paridad respecto de cuando no (segundo caso). No podemos olvidar que la paridad no es el final del camino, sino el comienzo (Varela, 2013).

De igual modo, hemos constatado en el análisis interno la existencia de una desigualdad dentro de la propia desigualdad. La mujer asume menos poder respecto a los hombres en el seno de los consistorios. Además, las áreas de las que se las responsabiliza son mayormente aquellas relativas al cuidado de terceras personas (Sanidad, Servicios Sociales) o de su propia promoción (Igualdad, Mujer, Integración), quedando reservadas para los hombres las áreas de mayor trascendencia (Hacienda, Urbanismo, Obras, Promoción económica...etc). Para mayor ahínco, la mujer promociona en términos porcentuales menos en política respecto de los varones, que son más tendentes a repetir de una legislatura a otra, reforzado en el caso de repetir en las alcaldías. Todo ello sin perder de vista el factor dimensional del municipio, dado que, a medida que tienen menos población, mayor es la brecha de género existente en su distribución municipal.

En cómputo, España, aunque mejora sus cifras progresivamente gracias al impulso de medidas legales como la cuota electoral de género, que favorecen el acceso igualitario a los cargos públicos, así como a una mayor concienciación existente en los propios partidos políticos que comienzan a adoptar medidas, no deja de demostrar que existe una evidente necesidad de hacer de la paridad y la igualdad de género un bien público, espejo de la sociedad, que consiga su ramificación hacia las esferas privadas. Ni las disposiciones legales, ni las cuotas orgánicas como medidas de discriminación positivas están siendo suficientes, aunque sí han sido favorables para que la mujer acceda a la administración pública. Sin embargo, ni unas ni otras harían falta si existiera una voluntad real en los propios partidos políticos (Sánchez, 2008).

Ciertamente las personas no somos meramente cifras, pero, en ocasiones, resulta imprescindible plasmar esta serie de datos para poner en antecedente a la sociedad ante un problema latente en nuestra democracia. Existen insuficientes trabajos que pongan el 
foco en la brecha de género todavía visible en el poder local. Es imprescindible no solo la elaboración cualquier estudio al respecto, sino, además, el abordaje de medidas para reducir unas cifras que todavía alarman.

\section{Bibliografía}

Cabo, Rosa (2002). Democracia paritaria y su jeto político feminista. Anales de la Cátedra Francisco Suárez, 36, pp. 29-44.

DAVIDSON-Schmich, Louise. K. (2006). Implementation of Political Party Gender Quotas. Evidence from the German Länder 1990-2000, Party Politics, 12 (2): 211-232.

GARcía, JuAn José (2011). El sexo excluido. Mujer y participación política. Psicología Política, $\mathrm{n}$-42, pp. 13-27.

SÁNCHEZ, GEMA (2008). El papel de las mujeres en la política española: el caso del PSOE. Estudios Feministas, Florianópoli, 16 (2), 440.

VALCÁRCEL, AMElia (1997). La política de las mujeres. Madrid: Cátedra.

VARELA, NuRIA (2013). Feminismo para principiantes. Barcelona: Ediciones B.

VERGE, TANIA (2006). Mujer y partidos políticos en España: las estrategias de los partidos y su impacto institucional, 1978-2004. Reis, 115/06, 165-196.

\section{Fuentes de datos}

Instituto de la Mujer.

Ministerio de Hacienda y Administraciones Públicas.

Ministerio del Interior.

MiYares, Alicia (2003). Democracia feminista. Madrid: Cátedra.

Resultados y conclusiones del informe de impacto de la Ley de Igualdad en las elecciones locales de 2007. http://www.femp.es/files/11-134-fichero/Informe\%20de $\% 20$ Igualdad.pdf 